\title{
CHARACTERIZATION OF LARGE GEM MODULE FOR THE TRACKER AT JLAB HALL A
}

\author{
F. Mammoliti ${ }^{1,2}$,a , V. Bellini ${ }^{1,2}$, E. Cisbani ${ }^{3,4}$, F. Librizzi ${ }^{2}$, P. Musico ${ }^{5}$, F. Noto ${ }^{1,2}$, R. Perrino ${ }^{6}$, L. Re ${ }^{1,2}$, and M.C. \\ Sutera $^{1,2}$ \\ ${ }^{1}$ Dipartimento di Fisica ed Astronomia, Università di Catania, via Santa Sofia 64, I-95123 Catania, Italy \\ 2 INFN - Sezione di Catania, via Santa Sofia 64, I-95123 Catania, Italy \\ ${ }^{3}$ INFN - Sezione di Roma La Sapienza, P.le Aldo Moro, 2 I-00185 Roma, Italy \\ ${ }^{4}$ Istituto Superiore di Sanità, viale Regina Elena 299, I-00161 Roma, Italy \\ ${ }^{5}$ INFN - Sezione di Genova, via Dodecaneso 33, I-16146 Genova, Italy \\ ${ }^{6}$ INFN - Sezione di Lecce, via Arnesano, I-73100 Lecce, Italy
}

\begin{abstract}
A new Large-Acceptance Forward Angle Spectrometer (Super BigBite) is under development at $\mathrm{JLab} / \mathrm{Hall} \mathrm{A}$ for the upcoming experiments in Hall A at Jefferson Lab where a longitudinally polarized electron beam of $11 \mathrm{GeV}$ is now available. This beam, combined with innovative polarized targets will provided luminosity up to $10^{39} /\left(\mathrm{s} \cdot \mathrm{cm}^{2}\right)$ opening exciting opportunities to investigate unexplored aspects of the inner structure of the nucleon. The tracker of this new apparatus is based on the Gas Electron Multiplier (GEM) technology, which has been chosen to optimize cost/performance, position resolution and to meet the high hit rate $\left(>1 \mathrm{MHz} / \mathrm{cm}^{2}\right)$.The first GEM detector modules, designed and built by the INFN Collaboration JLAB12, were tested at the DESY test beam facility in Hamburg, by using an electron beam with energy ranging from 2.0 to $6.0 \mathrm{GeV}$. In particular, three $40 \times 50 \mathrm{~cm}^{2}$ GEM chambers were equipped with a new implementation of the APV25 readout chip. Measurements were performed at different impact points and angles between the electron beam and the plane of the GEM chambers, with one large chamber in a solenoid magnetic field up to 500 Gauss. In this paper we present the technical features of the tracker and comment on the presently achieved performance.
\end{abstract}

\section{Introduction}

The Jefferson Laboratory (JLab) [1] is one of the most important experimental facility providing a multi $\mathrm{GeV}$, high intensity, longitudinally polarized, electron beam. Different relevant fields of fundamental physics are investigated at JLab: the origin of the quark and gluon confinement, the dynamics of the quarks and gluons in the nucleon and of the nucleon in the nucleus, the structure of the nuclei and the limits of the standard model. JLAB is undergoing a major upgrade of its CEBAF electron beam and experimental halls. In particular, CEBAF will deliver electron with energy up to $12 \mathrm{GeV}$ (twice the previous limit) with excellent intensity (up to $100 \mu A$ ) and longitudinal polarization (up to $85 \%$ ). In order to exploit the opportunities of the new beam, the equipments of the 3 existing experimental Halls are under upgrading. In particular members of Hall A collaboration are developing a new reconfigurable spectrometer, the Super BigBite (SBS [2], fig.1), featuring very forward angle (down to 7 degrees), large momentum (2-10 GeV/c) and angular acceptance (64 mrad), high rate capability $\left(1 \mathrm{MHz} / \mathrm{cm}^{2}\right)$ and very high luminosity environment (up to $10^{39} /\left(\mathrm{s} \cdot \mathrm{cm}^{2}\right)$ ). In its full configuration,

\footnotetext{
a e-mail: francesco.mammoliti@ct.infn.it
}

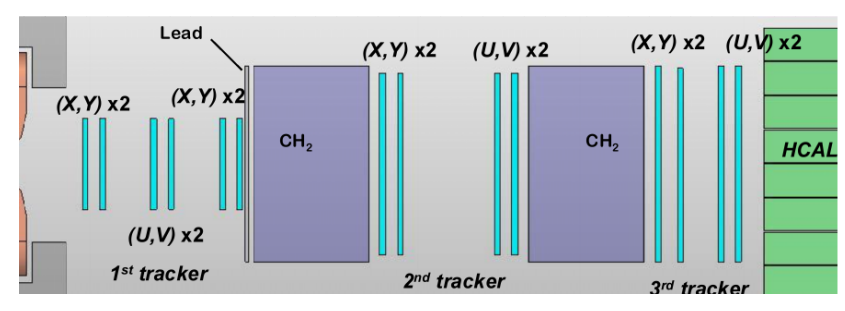

Figure 1. Schematic layout of the SBS Spectrometer: there are three stations with trackers.

the new spectrometer will consist of a dipole magnet with field integral up to $3 T$ (it will operate at about $2 T$ ), a primary charged particle tracker (first tracker), 2 identical proton polarimeters (made of a Carbon analyzer and large tracker), and an hadron calorimeter. SBS will initially serve 4 experiments [3] dedicated to the study of the nucleon structure in terms of elastic electromagnetic form factors at high 4-momentum transfer $Q^{2}$ up to $15 \mathrm{GeV}^{2}$ and of transverse momentum distributions of the quarks in the SIDIS (Semi Inclusive Deep Inelastic Scattering) region. The tracking systems of SBS will be mainly based on GEM chambers. The main features of the SBS tracker 
and of the GEM detector will be presented in the next section and, finally, we will discuss about the results of the test performed at DESY.

\section{SBS Tracker and GEM Detector}

The SBS tracking system is made of three stations. The primary (front) tracker, placed just after the dipole momentum analyzing magnet, will consist of six large area $\left(40 \times 150 \mathrm{~cm}^{2}\right)$ and high resolution $(\approx 70 \mu \mathrm{m})$ GEM chambers, for a total tracker length of about $50 \mathrm{~cm}$. Each chamber is made by 3 adjacent GEM modules of $40 \times 50 \mathrm{~cm}^{2}$ active rectangular area, for a total of 18 modules. It is designed to be capable to track accurately particles emerging from the electron scattering in a large background of soft photons $\left(\approx 0.5 \mathrm{MHz} / \mathrm{cm}^{2}\right)$ and MIPs $\left(\approx 0.2 \mathrm{MHz} / \mathrm{cm}^{2}\right)$. The primary tracking will be reinforced by combination with two small $\left(10 \times 20 \mathrm{~mm}^{2}\right)$ planes of silicon $\mu$ strips placed immediately after the target. The other stations are meant to track particles after a polarization analyzer wall and will require less accuracy. The primary tracker is under the responsibility of INFN groups. GEM technology [4] has been chosen to optimize cost/performance, position resolution and meet the high rate $\left(>1 \mathrm{MHz} / \mathrm{cm}^{2}\right)[5]$. The single module is made of 3 GEM foils and double layer $x / y$ strips readout with $400 \mu$ strip pitch (figure 2). The $8 \mathrm{~mm}$ wide mechanical frame incorporates high voltage feeding protection resistors and gas inlet/outlet holes. The signals from each triple GEM module are read out in two coordinates through COMPASS-like [6] strip conductors planes. The front-end electronics [7] (FE) for the $\approx 100 \mathrm{~K}$ channels of the tracker is based on the APV25 [8] chip, successfully used in the LHC experiment CMS. The APV25 is a serial output analogue ASIC running at $40 \mathrm{MHz}$. The FE cards, each with 128 channels, are placed around the GEM module. Custom backplanes are used to distribute power and control to the FE cards and to collect the analogue outputs.

In figure 3 a fully equipped $40 \times 50 \mathrm{~cm}^{2}$ GEM module setup under test at DESY is shown. The module is equipped with the APV25 electronics and 18 front-end cards are located behind the 4 rectangular backplanes that sit along the 4 sides of the module. During the test, a gas mixture of $\mathrm{Ar}(70 \%)$ and $\mathrm{CO}_{2}(30 \%)$ has been used and $\mathrm{HV}$ has been powered by the HV-GEM system [9] providing 7 independent HV levels. Moreover, precise tracking has been performed by small scintillators detector located before the GEM. The test was carried out in the T22 DESY Test electron/positron beam area [10]. The test beam is originated from the lepton synchrotron DESY II by converted bremsstrahlung on a carbon fiber target. The energy of the beam varies between about 1 and $6 \mathrm{GeV} / \mathrm{c}$ with typical intensity of 1000 particle $/\left(\mathrm{s} \cdot \mathrm{cm}^{2}\right)$ (divergence is about $2 \mathrm{mrad}$ ).

\section{Data Analysis and Discussion}

Preliminary results of data analysis performed on about 50 beam runs, obtained by using three GEM chambers
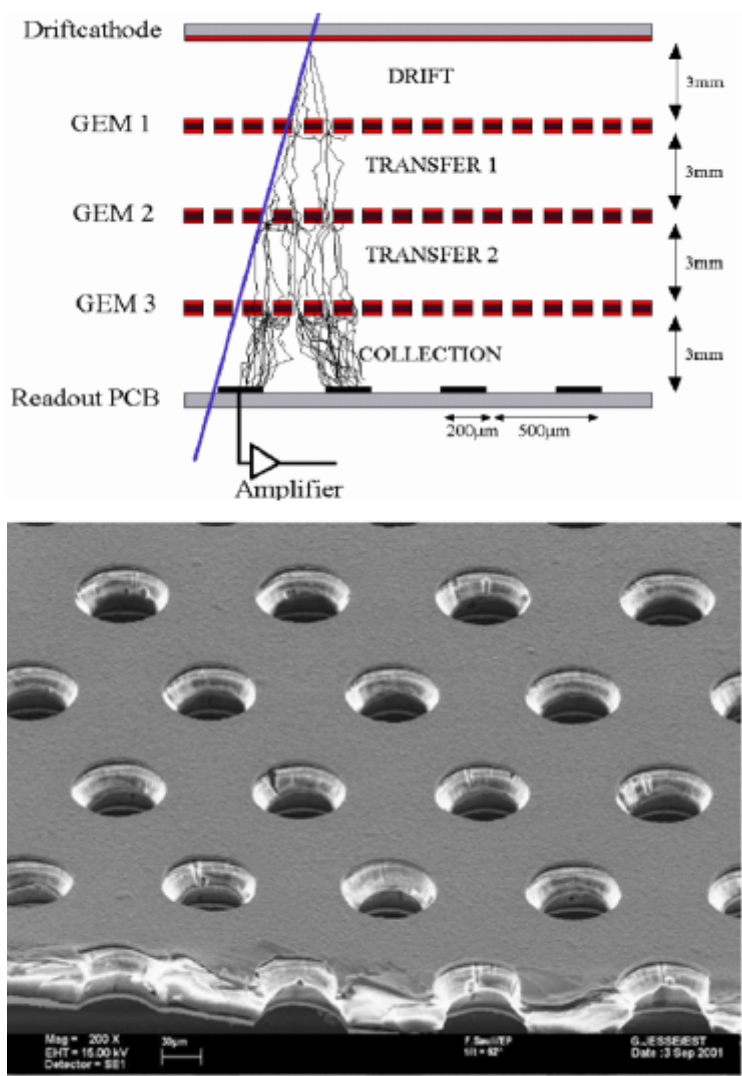

Figure 2. Top panel: schematic layout of the GEM Chamber (the total gain is about $10^{6}$ ). Bottom panel: image of a GEM foil obtained by an electron microscope (each hole has a biconical structure with a diameter of 70 and $50 \mu m$ in the wide and narrow area, respectively).

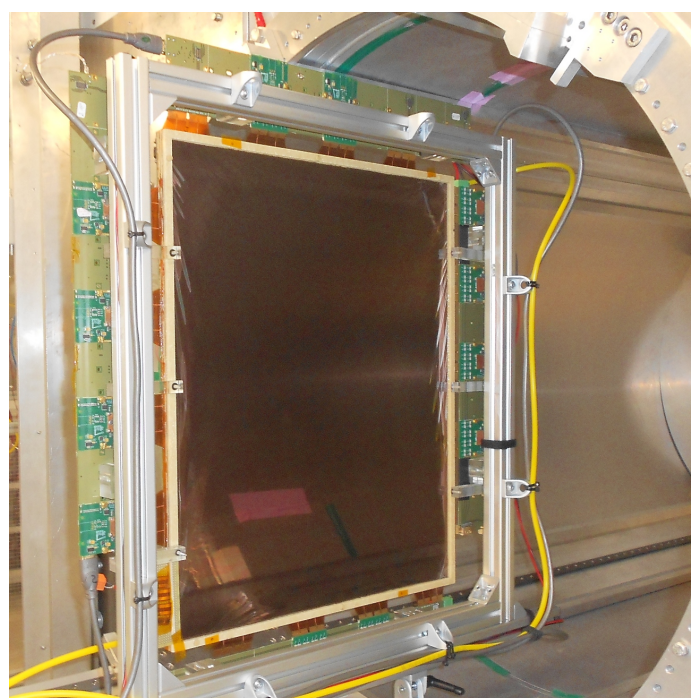

Figure 3. Fully equipped 40x50 $\mathrm{cm}^{2}$ GEM module setup under test at DESY. 


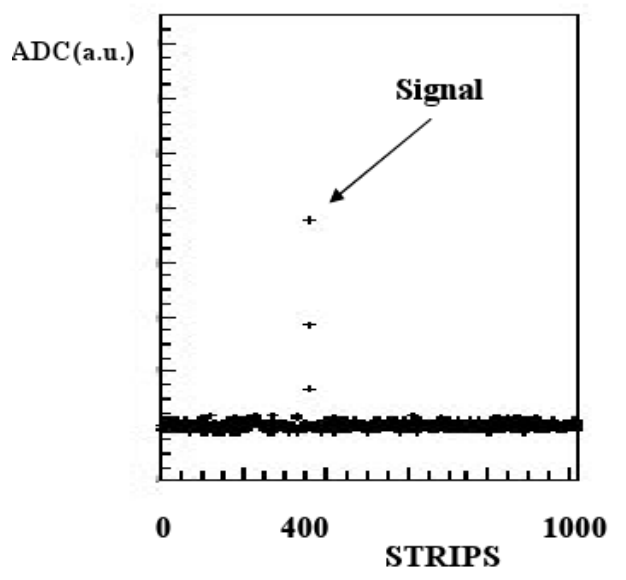

Figure 4. Single event signal in the $\mathrm{x}$-direction (the value of adc is given in arbitrary units).

with a dimension of $40 \times 50 \mathrm{~cm}^{2}$, are presented in this section. GEM chambers were readout by the APV electronics which were under development at the same time. Different configurations have been used during the test: energy of the electron beam (from 2.0 to $6.0 \mathrm{GeV}$ ), HV settings, angle between the beam and the plane of the chamber and position of the chamber with respect to the beam. Moreover, in order to measure pedestals, without beam runs were acquired. Common noise suppression, pedestal subtraction and hot channels masking are included in the standard analysis process. A single signal in the $\mathrm{x}$-direction is shown in figure 4: it is clearly visible at about strip \#380 and it is obtained after the pedestal subtraction. By using APV 25 chips, it is possible to record different parts of the signal (every $25 \mathrm{~ns}$ ), event by event. The shape of the signal was fitted (not shown) by using the formula (1):

$$
A\left[1-\exp \left(-\frac{t-t_{0}}{\tau_{1}}\right)\right] \cdot \exp \left(-\frac{t-t_{0}}{\tau_{2}}\right)
$$

in which $\tau_{1}$ and $\tau_{2}$ are the slope and falling time of the signal, respectively, $t_{0}$ is the start time and $\mathrm{A}$ is the signal amplitude. In particular, we have found a start time spread lower than $5 \mathrm{nsec}$ (see figure 5).

Adjacent firing strips are grouped in clusters which number was evaluated event by event in the $\mathrm{y}$ - vs $\mathrm{x}$ - direction (figure 6-top). It is evident that a low number of clusters is present in a single event and, moreover, there are only few strips in each cluster (not shown). In both cases, distributions are consistent with the data from COMPASS GEM characterization [11]. The percentage of events with a minimum of one cluster is about $96 \%$ for all 3 chambers used during the test. The beam profile is shown in figure 6-bottom and a value of 4-6 $\mathrm{mm}$, similar to the nominal value of the spot size, was found.

In order to select the single events of a run, we checked if there is a cluster on each chamber in the $\mathrm{x}$-direction (see figure 7). Each cluster provides the hit position $P_{n}\left(Z_{n}, X_{n}\right)$ and its uncertainty $\sigma_{n}$, where $\mathrm{n}$ is the index of the chamber.

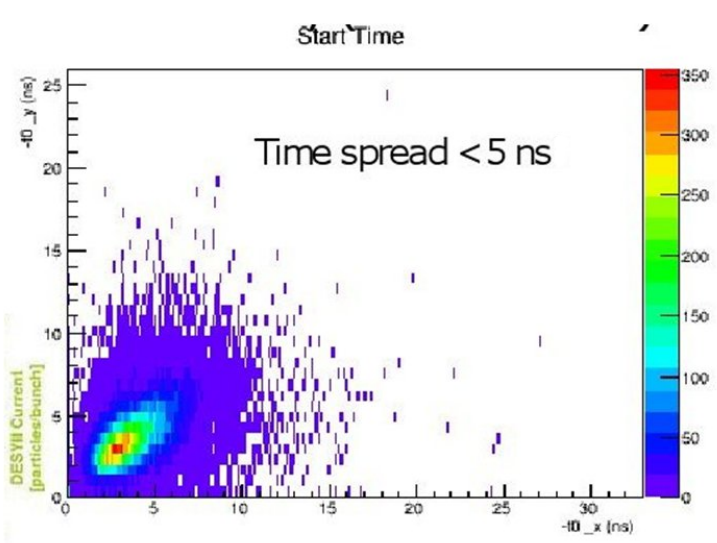

Figure 5. Start Time Spread (lower than $5 \mathrm{nsec}$ ).
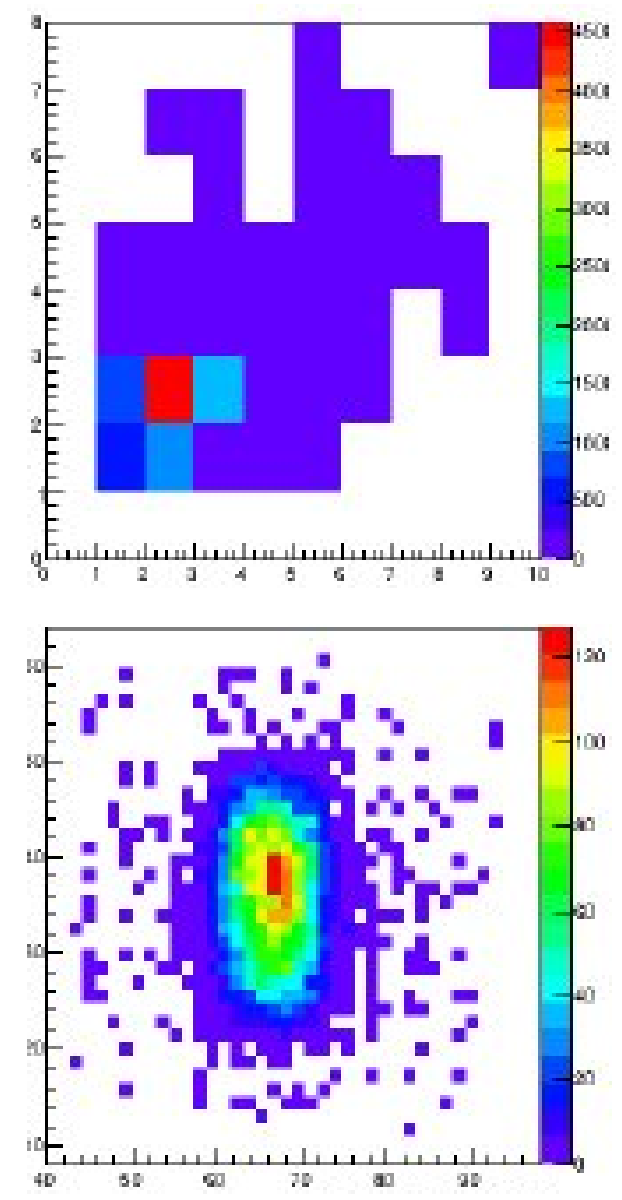

Figure 6. Top panel: number of clusters. Bottom panel: beam profile. 


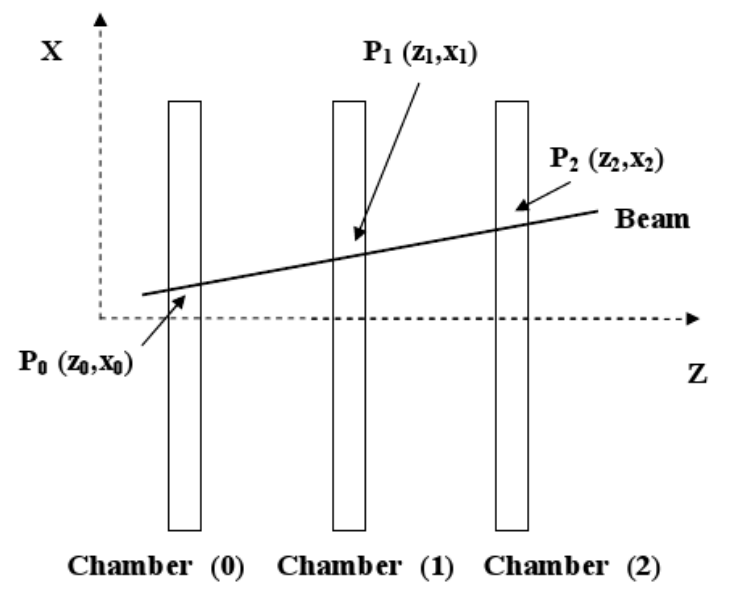

Figure 7. Schematic layout of the three GEM chambers during the test.

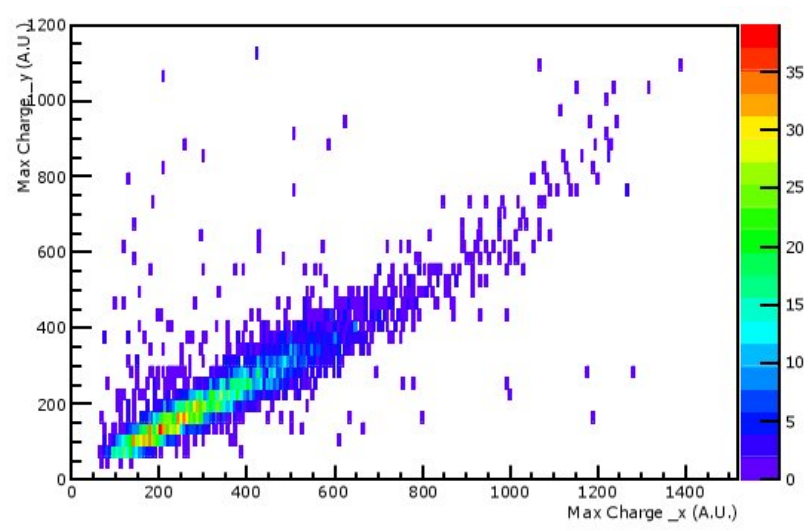

Figure 8. Charge correlation (a value of about $98 \%$ was found).

By using two points, for example $P_{1}\left(Z_{1}, X_{1}\right)$ and $P_{2}\left(Z_{2}, X_{2}\right)$, a straight line $\mathrm{x}=\mathrm{a} \cdot \mathrm{z}+\mathrm{b}$ is reconstructed in the $\mathrm{z}-\mathrm{x}$ twodimensional space ( $\mathrm{a}$ and $\mathrm{b}$ are obtained by a linear fit). Finally, we consider $P_{0}\left(Z_{0}, X_{0}\right)$ and if $\left|X_{0}-\mathrm{a} Z_{0}-\mathrm{b}\right|<\sigma_{0}$ than the signal of the three chambers belongs to the same particle otherwise the event is rejected. The ratio between the number of selected events and the total number of events in which a cluster was found on each chamber gives a percentage value of about $92 \%$. In figure 8 , the total charge collected in the $\mathrm{y}$ - vs $\mathrm{x}$ - direction is plotted and a value of about $98 \%$ for the correlation coefficient has been found.

\section{Conclusions}

The GEM chambers operated stably during the test and the results show reasonable indications about the detector performances. The main purpose of the test was to verify the overall functionality of the main solutions adopted in the first GEM modules under simple beam conditions. Both GEM hardware and readout electronics were under development and preliminary results on chamber efficiency and resolution were obtained. Further investigations about the test data are in progress.

\section{References}

[1] Thomas Jefferson National Accelerator Facility, www.jlab.org.

[2] The SBS project web site: http://hallaweb.jlab.org/12GeV/SuperBigBite/.

[3] $12 \mathrm{GeV}$ HallA upgrade web site: http://hallaweb.jlab.org/12GeV/.

[4] Sauli F., Nucl. Instr. Meth. A 386, 531 (1997).

[5] Bellini V. et al., JINST 7, C05013 (2012), proceedings of the MPGD 2012 conference.

[6] Ketzer B., Nucl. Instr. Meth. A 494, 142 (2002).

[7] Musico p. et al., 2011 IEEE Nuclear Science Symposium Conference Record.

[8] French M.J. et al., Nucl. Instrum. Meth. A 466, 359 (2002).

[9] $12 \mathrm{GeV}$ HallA upgrade web site: http://hallaweb.jlab.org/12GeV/.

[10] DESY Test Beams web site: http://adweb.desy.de/ testbeam/.

[11] Altunas C. et al., CERN-EP/2002-008, CERN, 21 January 2002. 